\title{
Neuropatologia da ataxia espinocerebelar tipo 3 (SCA3) - a doença de Machado-
}

\author{
Joseph \\ Neuropathology of spinocerebellar ataxia type 3 (SCA3) - Machado-Joseph disease \\ Neuropatología de la ataxia espinocerebelosa tipo 3 (SCA3) - Enfermedad de Machado-Joseph
}

Recebido: 19/02/2021 | Revisado: 28/02/2021 | Aceito: 03/03/2021 | Publicado: 11/03/2021

Rodolfo Lopes Vaz

ORCID: https://orcid.org/0000-0002-1453-8835 Centro Universitário de Anápolis, Brasil

E-mail: rodolfo236vaz@gmail.com

Gabriela Ramos Ribeiro

ORCID: https://orcid.org/0000-0001-7036-6743 Centro Universitário de Anápolis, Brasil E-mail: gabi28rr@hotmail.com

Lara Gomes Nery

ORCID: https://orcid.org/0000-0001-7568-8987 Centro Universitário de Anápolis, Brasil E-mail: laragnery@gmail.com

Ana Cláudia Maia Mendonça da Costa

ORCID: https://orcid.org/0000-0003-2719-9446 Centro Universitário de Anápolis, Brasil E-mail: anacmmendonca@gmail.com Gustavo Silva Oliveira

ORCID: https://orcid.org/0000-0001-6486-2759 Centro Universitário de Anápolis, Brasil E-mail: gustavosilvaoliveiram@gmail.com

Jalsi Tacon Arruda

ORCID: https://orcid.org/0000-0001-7091-4850 Centro Universitário de Anápolis, Brasil E-mail: jalsitacon@gmail.com

\begin{abstract}
Resumo
A ataxia espinocerebelar tipo 3, também conhecida como doença de Machado-Joseph, é um distúrbio neurodegenerativo autossômico dominante, provocado por uma expansão da repetição CAG que codifica a glutamina no gene ATXN3. Afeta tanto homens como mulheres, com sintomas em geral tardios, surgindo por volta da segunda década de vida e piorando com o avançar da idade. Apesar de rara, é a forma mais comum de ataxia espinocerebelar e não há evidências de terapias capazes de promover a cura. Assim, o presente estudo reuniu informações para atualizar os conhecimentos sobre essa doença. Trata-se de uma revisão integrativa de literatura realizada nas bases de dados PUBMED, SciELO e BVS. A ataxia cerebelar progressiva é comum nesses pacientes. No espectro clínico inclui anormalidades oculomotoras, distúrbios do sono, síndrome piramidal, sinais extrapiramidais e neuropatia periférica. Esses sintomas neurológicos são expressos por efeito da perda neuronal em regiões específicas do cerebelo, gânglios da base, tronco encefálico e medula espinhal. Pode ocorrer ansiedade, depressão e alto nível de fadiga. Ainda não há um tratamento eficaz que seja capaz de modificar o curso natural dessa doença. Os estudos mais recentes têm como alvo terapêutico o silenciamento gênico e a interceptação dos efeitos tóxicos provocados pelas proteínas produzidas. Terapias sintomáticas e fisioterapia tem sido realizada para melhora da qualidade de vida. Conhecer as características clínicas da doença de Machado-Joseph, que é uma condição grave, incurável, altamente debilitante e capaz de gerar uma alta carga de sofrimento nos seus portadores é importante na atuação do neurologista.
\end{abstract}

Palavras-chave: Neurologia; Quadro clínico; Tratamento; Qualidade de vida.

\begin{abstract}
Spinocerebellar ataxia type 3, also known as Machado-Joseph disease, is an autosomal dominant neurodegenerative disorder, caused by an expansion of the CAG repeat that encodes glutamine in the ATXN3 gene. It affects both men and women, with symptoms that are usually late, appearing around the second decade of life and worsening with advancing age. Although rare, it is the most common form of spinocerebellar ataxia and there is no evidence of therapies capable of promoting a cure. Thus, the present study gathered information to update knowledge about this disease. This is an integrative literature review carried out in the PUBMED, SciELO and BVS databases. Progressive cerebellar ataxia is common in these patients. In the clinical spectrum it includes oculomotor abnormalities, sleep disorders, pyramidal syndrome, extrapyramidal signs and peripheral neuropathy. These neurological symptoms are expressed by the effect
\end{abstract}


of neuronal loss in specific regions of the cerebellum, basal ganglia, brain stem and spinal cord. Anxiety, depression and a high level of fatigue can occur. There is still no effective treatment that can change the natural course of this disease. The most recent studies have the therapeutic target of gene silencing and the interception of toxic effects caused by the proteins produced. Symptomatic therapies and physical therapy have been performed to improve quality of life. Knowing the clinical characteristics of Machado-Joseph disease, which is a serious, incurable, highly debilitating condition and capable of generating a high burden of suffering in its patients, is important in the performance of the neurologist.

Keywords: Neurology; Clinical condition; Treatment; Quality of life.

\section{Resumen}

La ataxia espinocerebelosa tipo 3, también conocida como enfermedad de Machado-Joseph, es un trastorno neurodegenerativo autosómico dominante, causado por una expansión de la repetición CAG que codifica la glutamina en el gen $A T X N 3$. Afecta tanto a hombres como a mujeres, con síntomas que suelen ser tardíos, apareciendo alrededor de la segunda década de la vida y empeorando con la edad. Aunque es poco común, es la forma más común de ataxia espinocerebelosa y no hay evidencia de terapias capaces de promover una cura. Así, el presente estudio recopiló información para actualizar los conocimientos sobre esta enfermedad. Se trata de una revisión integradora de la literatura realizada en las bases de datos PUBMED, SciELO y BVS. La ataxia cerebelosa progresiva es común en estos pacientes. En el espectro clínico incluye anomalías oculomotoras, trastornos del sueño, síndrome piramidal, signos extrapiramidales y neuropatía periférica. Estos síntomas neurológicos se expresan por el efecto de la pérdida neuronal en regiones específicas del cerebelo, los ganglios basales, el tronco encefálico y la médula espinal. Pueden producirse ansiedad, depresión y un alto nivel de fatiga. Todavía no existe un tratamiento eficaz que pueda cambiar el curso natural de esta enfermedad. Los estudios más recientes tienen como objetivo terapéutico el silenciamiento de genes y la interceptación de efectos tóxicos provocados por las proteínas producidas. Se han realizado terapias sintomáticas y fisioterapia para mejorar la calidad de vida. Conocer las características clínicas de la enfermedad de Machado-Joseph, que es una condición grave, incurable, altamente debilitante y capaz de generar una alta carga de sufrimiento en sus pacientes, es importante en la actuación del neurólogo.

Palabras clave: Neurología; Cuadro clínico; Tratamiento; Calidad de vida.

\section{Introdução}

As ataxias são distúrbios neurológicos que afetam o controle motor. As ataxias espinocerebelares (SCA Spinocerebellar Ataxia) compõe um grupo heterogêneo de doenças progressivas, autossômicas dominantes. A característica clínica mais evidente é a perda de equilíbrio e coordenação, acompanhada por fala arrastada; de início tardio mais frequentemente na vida adulta (Koeppen, 2018). As ataxias são agrupadas geneticamente como SCA de expansão de repetição, como a SCA3/Doença de Machado-Joseph (DMJ), e SCA raras causadas por mutações não repetidas, como a SCA5. A maioria das mutações causa danos aos neurônios de Purkinje cerebelares que apresentam atrofia cerebelar consecutiva, embora alguns tipos de ataxia afetem levemente os neurônios de Purkinje. Outras partes do sistema nervoso, como a medula espinhal, gânglios da base e núcleos pontinos no tronco encefálico, podem estar envolvidos (Klockgether et al., 2019).

A ataxia espinocerebelar tipo 3 (SCA3), conhecida como a doença de Machado-Joseph, é a ataxia autossômica dominante mais comum. Integra um grande grupo de doenças conhecidas como poliglutamínicas (polyQ) (Duarte-Silva \& Maciel, 2018). Ocorre uma mutação do gene ATXN3 provocada por uma expansão da repetição CAG que codifica a glutamina no gene e ocasiona a SCA3/DMJ (Hou et al., 2018). O produto proteico do gene mutado, a ataxina-3, é uma enzima expandida de forma aberrante com ação desubiquitinante, que remove e desmonta cadeias de ubiquitina de substratos específicos, capaz de agregar e gerar espécies tóxicas que perturbam vários sistemas celulares, incluindo autofagia, proteostase, transcrição, função mitocondrial e sinalização celular (Matos et al., 2019; Dantuma; Herzog, 2020). Essa causa monogênica simples contrasta com a complexidade dos mecanismos patogênicos que atualmente são admitidos como responsáveis pela disfunção neuronal e morte. E o dano estrutural começa na medula espinhal, pedúnculos cerebelares e na substância negra, progredindo para áreas encefálicas a longo prazo (Rezende et al., 2018). Partindo desses conhecimentos fundamentais sobre a SCA3/Doença de Machado-Joseph, o presente estudo reuniu informações para atualizar os conhecimentos sobre essa doença. 


\section{Metodologia}

Trata-se de uma revisão integrativa operacionalizada nas seguintes etapas: definição de uma questão norteadora de pesquisa; determinação dos critérios de inclusão e exclusão; seleção dos artigos; análise dos artigos selecionados, e por fim, a apresentação dos resultados obtidos. Uma revisão integrativa de literatura permite a síntese de diversos estudos publicados, possibilitando conclusões a respeito de uma área específica (Pereira et al., 2018). As buscas por artigos foram realizadas nas bases de dados: National Library of Medicine and National Institutes of Health (PubMed), Scientific Electronic Library Online (SciELO), Biblioteca Virtual em Saúde (BVS). Foram utilizados os seguintes Descritores em Ciências da Saúde, que constam na plataforma DeCS da Biblioteca Virtual de Saúde (BVS): Doença de Machado-Joseph, Ataxia espinocerebelar e Melhoria de qualidade, que foram utilizados em combinação aos termos "Quadro clínico e Tratamento" para ampliar as buscas, com auxílio de operadores Booleanos (and/or), em português, inglês e espanhol. Cada busca foi feita com os descritores combinados de maneira que um deles fosse relacionado à patologia (ex.: "Ataxia espinocerebelar"; "Doença de Machado-Joseph") e outro à terapêutica (ex.: "tratamento"; "melhoria de qualidade"). A busca foi complementada pelas referências citadas nas publicações encontradas.

Os critérios de inclusão foram: artigos originais ou de revisão, disponíveis na íntegra, que investigaram a ataxia espinocerebelar; publicados no período de 2018 a 2020; sem restrição de idioma de publicação. Foram excluídos estudos que não investigassem o tema proposto, artigos de opinião, carta ao editor, e que não atendessem aos critérios de inclusão descritos. A primeira etapa foi identificar se os estudos preenchiam os critérios de inclusão, passando por uma seleção inicial das publicações realizada com leitura do título e resumo disponível para verificar se enquadravam na temática. Após essa etapa, os estudos previamente selecionados foram lidos na íntegra, empregando os critérios de inclusão e exclusão, para análise e obtenção das informações necessárias para a construção do presente estudo. Caso houvesse discordância entre os avaliadores sobre os critérios analisados, foi realizada discussão específica sobre o artigo em questão, confrontando ideias com base nos critérios adotados para a revisão. A coleta de dados foi realizada entre março e dezembro de 2020. Os dados foram extraídos dos estudos selecionados para realizar a discussão das implicações para atuação clínica e pesquisas futuras.

\section{Resultados e Discussão}

O epônimo Doença de Machado-Joseph homenageia as primeiras famílias descritas com sintomas de ataxia progressiva, disartria, sinais piramidais iniciando por volta dos 40 anos de idade, herdada de modo autossômico dominante com parentesco na Ilha de Açores em Portugal. A primeira descrição da DMJ foi feita no Brasil em 1988. Embora rara a ataxia espinocerebelar tipo 3 é a mais comum, com distribuição geográfica distinta, atingindo picos de prevalência em algumas regiões do Brasil, Portugal e China (Koeppen, 2018; Mendonça et al., 2018; Klockgether et al., 2019).

A mutação causa uma expansão no número de repetições de trinucleotídios CAG (que codifica o aminoácido glutamina) no alelo do gene ataxina-3 (ATXN3 14q32.1), acima de 53 repetições (CAGexp). A sequência CAG está contida no éxon 10 do gene, que apresenta 11 éxons, e o alelo normal apresenta cerca de 12 a 43 repetições de CAG (OMIN-109150). Uma CAGexp em heterozigose é suficiente para causar a condição. O exame padrão-ouro para o diagnóstico da doença é a avaliação molecular que confirma a expansão do códon CAG (de Mattos et al., 2019). Estudos indicam uma forte correlação entre o número de repetições e a gravidade dos sintomas (Hou et al., 2018; Lima \& Raposo, 2018; Rezende et al., 2018). Quanto maior o número de repetições, mais graves são os sintomas e, ainda, quanto maior o número de repetições, mais cedo os sintomas se manifestam. Todavia, pouco ainda pode ser explicado apenas pela variação no tamanho da expansão do gene (Yang et al., 2020).

Por ser uma alteração autossômica dominante, basta possuir apenas um alelo alterado do gene para ser portador da SCA3/DMJ. Assim, o risco de transmissão genética é de 50\% considerando apenas um genitor afetado, e não há distinção em relação ao sexo. Além disso, a região CAGexp é instável durante as divisões celulares, e durante a meiose paterna pode aumentar 
consideravelmente explicando o fenômeno de antecipação dos sintomas característicos da SCA3/DMJ (de Mattos et al., 2019).

\subsection{Neuropatia}

A SCA3/DMJ afeta os neurônios do cerebelo devido a mutação herdada, os portadores sofrem com essa disfunção neurológica de coordenação motora, que pode afetar atividades fundamentais como o olhar, a fala, a marcha e o equilíbrio. Contudo, sabe-se que lesões na substância negra e núcleo denteado do cerebelo carregam o peso da doença, pois são responsáveis pelos fenótipos parkinsonianos e atáxicos. Distúrbios complexos da motilidade ocular e fraqueza da língua são provocados por lesões em núcleos motores no tronco cerebral. Anormalidades dos gânglios da base, medula espinhal, tálamo, gânglios da raiz dorsal e nervos periféricos sensoriais são variáveis. No entanto, a atrofia da base da ponte é comum, e os corpos de inclusão intranuclear neuronais positivos para poliglutamina são facilmente encontrados (Lima \& Raposo, 2018).

No cerebelo todas as camadas do córtex permanecem normais, porém o núcleo denteado exibe perda neuronal e uma proliferação peculiar de terminais sinápticos denominada de regeneração grumosa. No nível espinhal, os gânglios da raiz dorsal revelam proliferação de células satélite, destruição neuronal ativa e nódulos residuais. Já a medula espinhal apresenta perda total ou parcial de neurônios nos núcleos dorsais, atrofia das células do corno anterior e degeneração variável do trato longo (Koeppen, 2018).

A ataxia cerebelar progressiva apresenta um amplo espectro clínico. Entre os sintomas observados incluem síndrome piramidal, neuropatia periférica, anormalidades oculomotoras, sinais extrapiramidais e distúrbios do sono. Estudos de neuroimagem como por ressonância magnética estrutural (MRI - Magnetic Resonance Image), imagem por tensor de difusão (DTI - Diffusion Tensor Imaging), e espectroscopia por ressonância magnética (MRS - Magnetic Resonance Spectroscopy), podem ser úteis caracterizando diferenças estruturais e funcionais em subgrupos específicos de pacientes com DMJ (Mendonça et al., 2018).

Com base na presença/ausência de sinais extrapiramidais importantes e de sinais periféricos, foram definidos os subtipos clínicos (Matos et al., 2019). Dentre esses subtipos o tipo 2 é o mais frequente, seguido respectivamente dos tipos 3, 1, 4 e 5 . O tipo 1 é de início precoce, normalmente antes dos 20 anos, podendo progredir rapidamente com sinais piramidais proeminentes (rigidez e espasticidade) e características extrapiramidais (bradicinesia e distonia), bem como ataxia. $\mathrm{O}$ tipo 2 aparece numa idade de início moderada (20-50 anos) com ataxia cerebelar, sinais piramidais e oftalmoplegia externa progressiva. Já o tipo 3 tem início tardio (40-75 anos), e aparece com ataxia e sinais periféricos (neuropatia motora e atrofia muscular). O tipo 4 é caracterizado por parkinsonismo associado a outras características clínicas. E, por fim, mais recentemente o tipo 5 foi proposto para sinais clínicos de uma paraplegia espástica pura (Hou et al., 2018).

A proteína ataxina 3 é observada tanto no citoplasma como no núcleo das células participando do sistema ubiquitinaproteassoma. Ocorre a formação de neuroinclusões ou agregados intranucleares, marcadores patológicos na SCA3/DMJ, com efeito tóxico direto para a célula, com neurodegeneração predominante do cerebelo e núcleos do tronco cerebral (Dantuma; Herzog, 2020). Essas neuroinclusões incluem ubiquitina, atxn3, tratos de poliQ e outros fatores de transcrição. Ainda se observa grânulos citoplasmáticos imunomarcados com um anticorpo monoclonal contra PoliQ (1C2) correspondente a lisossomos, indicando que vias de autofagocitose para degradar a proteína ATXN3 mutada (Saute \& Jardim, 2018).

Avaliações da progressão da SCA3/DMJ são realizadas acompanhando as taxas de piora neurológica obtidas com a aplicação de escalas clínicas como: a International Cooperative Ataxia Rating Scale (ICARS), Scale for the Assesment and Rating of Ataxia (SARA), Neurological Examination Score for Spinocerebellar Ataxia (NESSCA), e a Composite Cerebellar Functional Score (CCSF) (Soong \& Morrison, 2018). Esses estudos mediram Escalas Clínicas para medir a progressão da doença, como também, para apontar os desfechos principais dentro do curso natural.

Estudos na população chinesa com SCA3/DMJ indicam que a maioria deles apresentaram sinais extracerebelares, sendo 
a rigidez o sinal mais comum (Yuan et al., 2019). Além disso, apresentaram altas frequências de ansiedade, depressão, fadiga e qualidade do sono prejudicada. A gravidade da depressão e ansiedade foi correlacionada ao comprimento da repetição CAG (Lin et al., 2018). A qualidade do sono foi associada com fadiga e gravidade da ataxia, mas não com sonolência diurna excessiva e condições psiquiátricas. Vários fatores, incluindo o comprimento da repetição CAG, idade de início, sono insatisfatório e depressão são preditores de fadiga nestes pacientes (Yuan et al., 2019).

A ataxia espinocerebelar tipo 3 exibe sintomas neuropsicológicos comuns, como depressão. Acredita-se que a depressão é resultado da deficiência motora causada pela ataxia, e impacta de forma significativa sobre a gravidade da doença. Esses achados sugerem que a depressão faz parte da neurodegeneração e necessita intervenções durante o atendimento dos pacientes (Lin et al., 2018).

Pacientes com SCA3/DMJ apresentam um nível de fadiga significativamente maior, sendo que a sonolência diurna e a gravidade atáxica são fatores de risco associados a fadiga. Estudos sugerem que exista uma relação bidirecional entre ataxia e fadiga. No entanto, os mecanismos fisiológicos para essa conexão permanecem desconhecidos e são considerados multifatoriais. Anormalidades do transportador de dopamina em pacientes com SCA3/DMJ foram descritas nestes casos. Ainda assim, estudos observaram dano microestrutural generalizado a substância branca no tálamo, substância branca cerebral bilateral e nos lobos frontal e temporal. Portanto, especula-se que anormalidades no transportador de dopamina e na substância branca do cérebro podem contribuir para a fadiga central observada em pacientes com SCA3/DMJ. A fadiga periférica observada pode ser devido a neuropatia periférica, uma vez que sintomas como cãibras musculares, fraqueza, dormência, perda muscular, dor neuropática e distúrbio do metabolismo energético do músculo esquelético são comuns (Yang et al., 2020).

\subsection{Tratamento}

Ainda não foi estabelecido nenhum tratamento específico que beneficie funcionalmente os pacientes, no entanto, há terapias que melhoram a sintomatologia. Por ser uma doença neurodegenerativa, o prognóstico é ruim, e comumente, com a evolução dos sinais e sintomas.

A influência da fadiga na gravidade atáxica destaca a necessidade de prevenção, sendo um sintoma não motor comum na SCA3/DMJ e outras doenças neurodegenerativas, como a doença de Parkinson (DP) e a esclerose lateral amiotrófica (ELA). Contudo, até agora, um tratamento ainda não foi relatado. Existe tratamento padronizado para fadiga em pacientes com DP, incluindo um regime de medicação balanceado visando transtorno do movimento (por exemplo, agentes dopaminérgicos) e depressão (como psicoestimulantes e antidepressivos) combinados com um programa de reabilitação (terapia cognitivocomportamental e exercícios graduais) (Lin et al., 2018). Já em relação a ELA, o manejo farmacológico (modafinil) e não farmacológico (exercício respiratório e estimulação magnética transcraniana repetitiva) pode ser uma opção de tratamento possível. Tais evidências indicam que a combinação apropriada de tratamentos farmacológicos e não farmacológicos pode ser opções promissoras para aliviar a fadiga e a gravidade da ataxia em pacientes com SCA3/DMJ (Yang et al., 2020).

Diversas pesquisas expandiram muito a compreensão da patobiologia de muitas SCA, revelando que ocorrem por meio de mecanismos interrelacionados (incluindo proteotoxicidade, toxicidade do RNA e disfunção do canal iônico), o que levou a identificação de novos alvos terapêuticos. No entanto, o desenvolvimento de terapias eficazes é dificultado pela heterogeneidade das SCA, já que abordagens terapêuticas específicas podem ser necessárias para cada tipo da doença (Klockgether et al., 2019).

Estudos de neuroimagem em indivíduos portadores da SCA3/DMJ sugerem que a disfunção do sistema nervoso começa muitos anos antes do início de quaisquer sintomas detectáveis. O desenvolvimento de intervenções terapêuticas torna-se de grande importância, não apenas para retardar a progressão da doença manifestada, mas também para retardar ou, idealmente, prevenir seu início (de Mattos et al., 2019).

Os alvos terapêuticos potenciais para DMJ e polyQ podem ser divididos em (i) aqueles que visam as próprias proteínas 
polyQ, nomeadamente silenciamento genético, tentativas de aumentar a degradação da proteína mutante ou inibição/prevenção da agregação; e (ii) aqueles que interceptam os efeitos tóxicos a jusante das proteínas polyQ, como disfunção mitocondrial e estresse oxidativo, anormalidades transcricionais, deficiência de UPS (sistema ubiquitina-proteassomo), excitotoxicidade ou ativação de morte celular. Até o momento, ainda não há um tratamento eficaz para DMJ e outras doenças de polyQ (DuarteSilva \& Maciel, 2018).

O silenciamento da proteína patogênica, o bloqueio da agregação, a inibição do processamento proteolítico tóxico e a neutralização das disfunções dos sistemas celulares afetados produziram resultados promissores em estudos com modelos celulares e animais (Matos et al., 2019). Compostos modificadores da doença começaram a ser testados no contexto de ensaios clínicos e o sucesso depende da sensibilidade dos métodos usados para medir benefícios terapêuticos sutis. Estudos propõem uma bateria de medidas de resultados potenciais, incluindo biomarcadores moleculares (MB), que ainda precisam ser identificados. Esses biomarcadores são testados em pacientes SCA por expressão gênica, utilizando complementariedade com outros marcadores clínicos e de imagem para rastrear totalmente a DMJ, desde sua fase pré-atáxica até o estágio de doença (Lima \& Raposo, 2018).

Pesquisas mais atuais com exossoma se mostraram bastante promissoras. Os Exossomos são vesículas minúsculas (40150nm), contendo RNA, DNA e proteínas secretadas por todas as células vivas, que podem ser detectadas em vários fluidos corporais. A identificação dessas moléculas é importante não só para o diagnóstico, como também para esclarecimento clínico da ataxia. O mir-7014 exossômico é um candidato a biomarcador significativo da SCA3/DMJ, que indica a classificação clínica e a idade de início. Ao correlacionar com os demais índices clínicos relacionados a pontuação da escala para avaliação e classificação da ataxia SARA (Scale for the Assessment of Rating Ataxia), repetições CAG e duração, nenhuma correlação óbvia foi identificada. Estudos também levantam a possibilidade de o mir-7014 exossômico poder ser um regulador significativo de alguns genes relacionados com o processo dessa patogênese. Além disso, o biomarcador apresenta um potencial terapêutico, uma vez que os exossomos encapsulados com mir-7014 podem ser entregues a partir dos vasos periféricos dos pacientes prevenindo a progressão da doença (Hou et al., 2018).

Apenas alguns tratamentos foram avaliados em ensaios clínicos randomizados em pacientes com SCA3/DMJ, com falta de evidências para terapias modificadoras da doença e sintomáticas. Os principais resultados para futuros ensaios clínicos são as escalas: a Escala para Avaliação e Classificação da ataxia (SARA) é atualmente o instrumento de escolha para provar a eficácia dos tratamentos modificadores da doença ou sintomáticos contra a ataxia, a característica mais importante da doença (Saute \& Jardim, 2018).

Ainda não existe um tratamento eficaz para DMJ, as terapias sintomáticas são usadas para aliviar alguns dos sintomas clínicos e a fisioterapia é útil para melhorar a qualidade de vida do paciente (Souza et al., 2018). Vários ensaios clínicos foram realizados com diferentes medicamentos, como sulfametoxazol-trimetoprima, vareniclina e carbonato de lítio, mas os resultados desses ensaios foram negativos ou mostraram poucos benefícios (Mendonça et al., 2018). Como atualmente não há tratamento para retardar ou interromper as SCA, muitas levam a morte prematura. O atendimento clínico se concentra no manejo dos sintomas por meio da fisioterapia, terapia ocupacional e fonoaudiologia (Duarte-Silva \& Maciel, 2018; Marques et al., 2020).

A fisioterapia é capaz de melhorar a independência funcional dos pacientes promovendo ganhos sobre a marcha, equilíbrio e força muscular, redução da frequência de quedas, e diminuição dos efeitos sintomáticos (Souza et al., 2018). A intervenção fonoaudiológica impacta positivamente na qualidade de vida dos pacientes com DMJ que sofrem com disartria e disfagia, pois alguns demonstraram melhora nas bases motoras de fala, fonatória, ressonantal, respiratória, articulatória e a prosódia, além da diminuição dos sinais disfágicos. Na qualidade vocal pode diminuir a instabilidade e rouquidão, aumentar a intensidade vocal, melhorar a coordenação de palavras e frases por expiração, e melhorar a diadococinesia (Marques et al., 2020).

O exame molecular que confirma a expansão do códon CAG é o padrão-ouro para o diagnóstico da doença. No entanto, 
uma questão bastante pertinente é o acesso a testes preditivos por parte dos familiares de pacientes com SCA3/DMJ. Há implicações éticas e legais que devem ser levadas em consideração. Estes testes devem fazer parte do aconselhamento genético abrangente que auxilie na tomada de decisão diante dessa possibilidade da doença.

\section{Considerações Finais}

Em virtude dos dados analisados observa-se a necessidade de conhecer as características clínicas e patológicas da doença de Machado-Joseph. Essa desordem neurológica é uma condição grave, até o momento incurável, altamente debilitante gerando sofrimento aos portadores. Preocupar-se com o bem-estar e a qualidade de vida destes pacientes é parte fundamental do tratamento, principalmente quanto ao manejo dos sintomas. Contudo, mais estudos serão necessários, na área da biotecnologia genética, para melhorar não só a compreensão da fisiopatologia, como para desenvolver tratamentos mais eficazes.

\section{Referências}

Dantuma, N. P., \& Herzog, L. K. (2020). Machado-Joseph Disease: A Stress Combating Deubiquitylating Enzyme Changing Sides. Advances in experimental medicine and biology, 1233, 237-260. https://doi.org/10.1007/978-3-030-38266-7_10

Duarte-Silva, S., \& Maciel, P. (2018). Pharmacological Therapies for Machado-Joseph Disease. Advances in Experimental Medicine and Biology. 1049:369394. DOI: 10.1007/978-3-319-71779-1_19.

Hou, X., Gong, X., Zhang, L., Li, T., Yuan, H., Xie, Y., Peng, Y., Qiu, R., Xia, K., Tang, B., \& Jiang, H. (2019). Identification of a potential exosomal biomarker in spinocerebellar ataxia Type 3/Machado-Joseph disease. Epigenomics, 11(9), 1037-1056. https://doi.org/10.2217/epi-2019-0081

Klockgether T, Mariotti C, \& Paulson HL. (2019). Spinocerebellar ataxia. Nature reviews. Disease Primers. 5(1):24. 10.1038/s41572-019-0074-3.

Koeppen A. H. (2018). The Neuropathology of Spinocerebellar Ataxia Type 3/Machado-Joseph Disease. Advances in experimental medicine and biology, 1049, 233-241. https://doi.org/10.1007/978-3-319-71779-1_11

Lima, M., \& Raposo, M. (2018). Towards the Identification of Molecular Biomarkers of Spinocerebellar Ataxia Type 3 (SCA3)/Machado-Joseph Disease (MJD). Advances in experimental medicine and biology, 1049, 309-319. https://doi.org/10.1007/978-3-319-71779-1_16

Lin, M. T., Yang, J. S., Chen, P. P., Qian, M. Z., Lin, H. X., Chen, X. P., Shang, X. J., Wang, D. N., Chen, Y. C., Jiang, B., Chen, Y. J., Chen, W. J., Wang, N., \& Gan, S. R. (2018). Bidirectional Connections between Depression and Ataxia Severity in Spinocerebellar Ataxia Type 3 Patients. European neurology, 79(56), 266-271. https://doi.org/10.1159/000489398

Marques, Thames dos Santos, Furia, Cristina Lemos Barbosa, \& Lira, Juliana Onofre de. (2020). Atuação fonoaudiológica na doença de Machado-Joseph: relato de caso. Audiology - Communication Research, 25, e2264. https://doi.org/10.1590/2317-6431-2019-2264

Matos, C. A., de Almeida, L. P., \& Nóbrega, C. (2019). Machado-Joseph disease/spinocerebellar ataxia type 3: lessons from disease pathogenesis and clues into therapy. Journal of neurochemistry, 148(1), 8-28. https://doi.org/10.1111/jnc.14541

Mattos, E. P., Kolbe Musskopf, M., Bielefeldt Leotti, V., Saraiva-Pereira, M. L., \& Jardim, L. B. (2019). Genetic risk factors for modulation of age at onset in Machado-Joseph disease/spinocerebellar ataxia type 3: a systematic review and meta-analysis. Journal of neurology, neurosurgery, and psychiatry, 90(2), 203210. https://doi.org/10.1136/jnnp-2018-319200

Mendonça, N., França, M. C., Jr, Gonçalves, A. F., \& Januário, C. (2018). Clinical Features of Machado-Joseph Disease. Advances in experimental medicine and biology, 1049, 255-273. https://doi.org/10.1007/978-3-319-71779-1_13

Rezende, T., de Paiva, J., Martinez, A., Lopes-Cendes, I., Pedroso, J. L., Barsottini, O., Cendes, F., \& França, M. C., Jr (2018). Structural signature of SCA3: From presymptomatic to late disease stages. Annals of neurology, 84(3), 401-408. https://doi.org/10.1002/ana.25297

Saute, J., \& Jardim, L. B. (2018). Planning Future Clinical Trials for Machado-Joseph Disease. Advances in experimental medicine and biology, 1049, 321-348. https://doi.org/10.1007/978-3-319-71779-1_17

Soong, B. W., \& Morrison, P. J. (2018). Spinocerebellar ataxias. Handbook of clinical neurology, 155, 143-174. https://doi.org/10.1016/B978-0-444-64189$2.00010-\mathrm{X}$

Souza F. J, Oliveira H. G., Marreiro J. S., \& Miguel M. M. M. C. (2018). A abordagem fisioterapêutica na marcha da doença Machado Joseph. Rev Inic Cient Ext;1(Esp):148-54.

Yang, J. S., Xu, H. L., Chen, P. P., Sikandar, A., Qian, M. Z., Lin, H. X., Lin, M. T., Chen, W. J., Wang, N., Wu, H., \& Gan, S. R. (2020). Ataxic Severity Is Positively Correlated with Fatigue in Spinocerebellar Ataxia Type 3 Patients. Frontiers in neurology, 11, 266. https://doi.org/10.3389/fneur.2020.00266

Yuan, X., Ou, R., Hou, Y., Chen, X., Cao, B., Hu, X., \& Shang, H. (2019). Extra-Cerebellar Signs and Non-motor Features in Chinese Patients with Spinocerebellar Ataxia Type 3. Frontiers in neurology, 10, 110. https://doi.org/10.3389/fneur.2019.00110 